\title{
Combined optical- and acoustic- resolution photoacoustic microscopy based on an optical fiber bundle
}

Wenxin Xing, Lidai Wang, Konstantin Maslov, Lihong V. Wang

Wenxin Xing, Lidai Wang, Konstantin Maslov, Lihong V. Wang, "Combined optical- and acoustic-resolution photoacoustic microscopy based on an optical fiber bundle," Proc. SPIE 8581, Photons Plus Ultrasound: Imaging and Sensing 2013, 858142 (4 March 2013); doi: 10.1117/12.2005259 


\title{
Combined optical- and acoustic-resolution photoacoustic microscopy based on an optical fiber bundle \\ Wenxin Xing ${ }^{\text {a,b }}$, Lidai Wang ${ }^{a}$, Konstantin Maslov ${ }^{a}$, Lihong V. Wang *a,b \\ ${ }^{a}$ Optical Imaging Laboratory, Department of Biomedical Engineering, \\ ${ }^{\mathrm{b}}$ Preston M. Green Department of Electrical and Systems Engineering \\ Washington University in St. Louis, One Brookings Drive, St. Louis, Missouri 63130, USA \\ * Corresponding author: lhwang@wustl.edu
}

\begin{abstract}
Photoacoustic microscopy (PAM), whose spatial resolution and penetration depth are both scalable, has made great progress in recent years. According to their different lateral resolutions, PAM systems can be categorized into either optical-resolution (OR) PAM, with optical-diffraction-limited lateral resolution, or acoustic-resolution (AR) PAM, with acoustically limited resolution and a deeper maximum imaging depth. In this report, we present a combined OR and AR PAM system with resolutions of $2.2 \mu \mathrm{m}$ and $40 \mu \mathrm{m}$, respectively. Sharing most components between the OR and AR implementations, the system achieves separated illumination for OR and AR imaging by an optical fiber bundle through different channels, and two discrete lasers are used to provide either high-power energy for AR imaging or highrepetition-rate pulses for OR imaging. The design enables automatically co-registered OR and AR photoacoustic imaging in one single system, which extends the usability of current photoacoustic systems and simplifies the imaging procedure.
\end{abstract}

Keywords: Photoacoustic imaging, fiber bundle

\section{INTRODUCTION}

Due to its optical absorption contrast and high spatial resolution that is scalable with the maximum imaging depth, photoacoustic microscopy (PAM) has been successfully applied to in vivo imaging at scales from organelles to organs $[1,2]$. According to their different lateral resolutions, PAM systems can be categorized into either acoustic-resolution (AR) PAM or optical-resolution (OR) PAM. AR-PAM [3, 4], whose lateral resolution is determined by the acoustic focus, can achieve tens of microns lateral resolution with a maximum imaging depth of several millimeters. OR-PAM [5,6] has a tighter optical focus than acoustic focus and can achieve opticaldiffraction-limited lateral resolution with a maximum imaging depth of $\sim 1.2 \mathrm{~mm}$ [7]. With these complementary characteristics, AR- and OR-PAM systems have been used in tandem to image samples in many applications $[8,9]$. However, since the systems share similar optical and ultrasonic components and designs, it is advantageous to combine them, which would facilitate either sequential or simultaneous operation, yielding automatically coregistered images and also reducing system cost. Here, we present a method to combine OR- and AR-PAM systems based on an optical fiber bundle.

\section{SYSTEM DESIGN}

A schematic of the system setup is shown in Fig. 1. A high-repetition-rate laser (SPOT 10-200-532, Elforlight) provides photoacoustic excitation for OR imaging, whereas a high-power laser (Cobra, Sirah) pumped by a $\mathrm{Nd}$ :YLF laser provides photoacoustic excitation for AR imaging. The high-repetition-rate laser operates at $532 \mathrm{~nm}$, near the 530-nm isosbestic absorption wavelength of oxy- and deoxy-hemoglobin, and it delivers light energy on the tissue surface up to $0.1 \mu \mathrm{J} / \mathrm{pulse}$ at $20 \mathrm{kHz}$. Because the high-power laser cannot operate at $532 \mathrm{~nm}$, it is tuned to another isosbestic point at $570 \mathrm{~nm}$, where hemoglobin has comparable optical absorption with hemoglobin at $532 \mathrm{~nm}$, and the delivered light energy on the tissue surface is up to $60 \mu \mathrm{J} / \mathrm{pulse}$ at $2 \mathrm{kHz}$. Emerging from the lasers, both beams are vertically polarized. The OR beam is focused by a condenser lens (LA1214-A, Thorlabs), then spatially filtered by a $25-\mu \mathrm{m}$-diameter pinhole. The AR beam passes through a beam expander (OL1 and OL2 ) with 1.5X magnification, and then becomes horizontally polarized by reflection off mirror M. The two perpendicular laser beams are combined by a polarizing beamsplitter cube (PBS201, Thorlabs). Because of the

Photons Plus Ultrasound: Imaging and Sensing 2013, edited by Alexander A. Oraevsky, Lihong V. Wang, Proc. of SPIE Vol. 8581, 858142 - (C) 2013 SPIE · CCC code: 1605-7422/13/\$18 - doi: 10.1117/12.2005259 
(a)
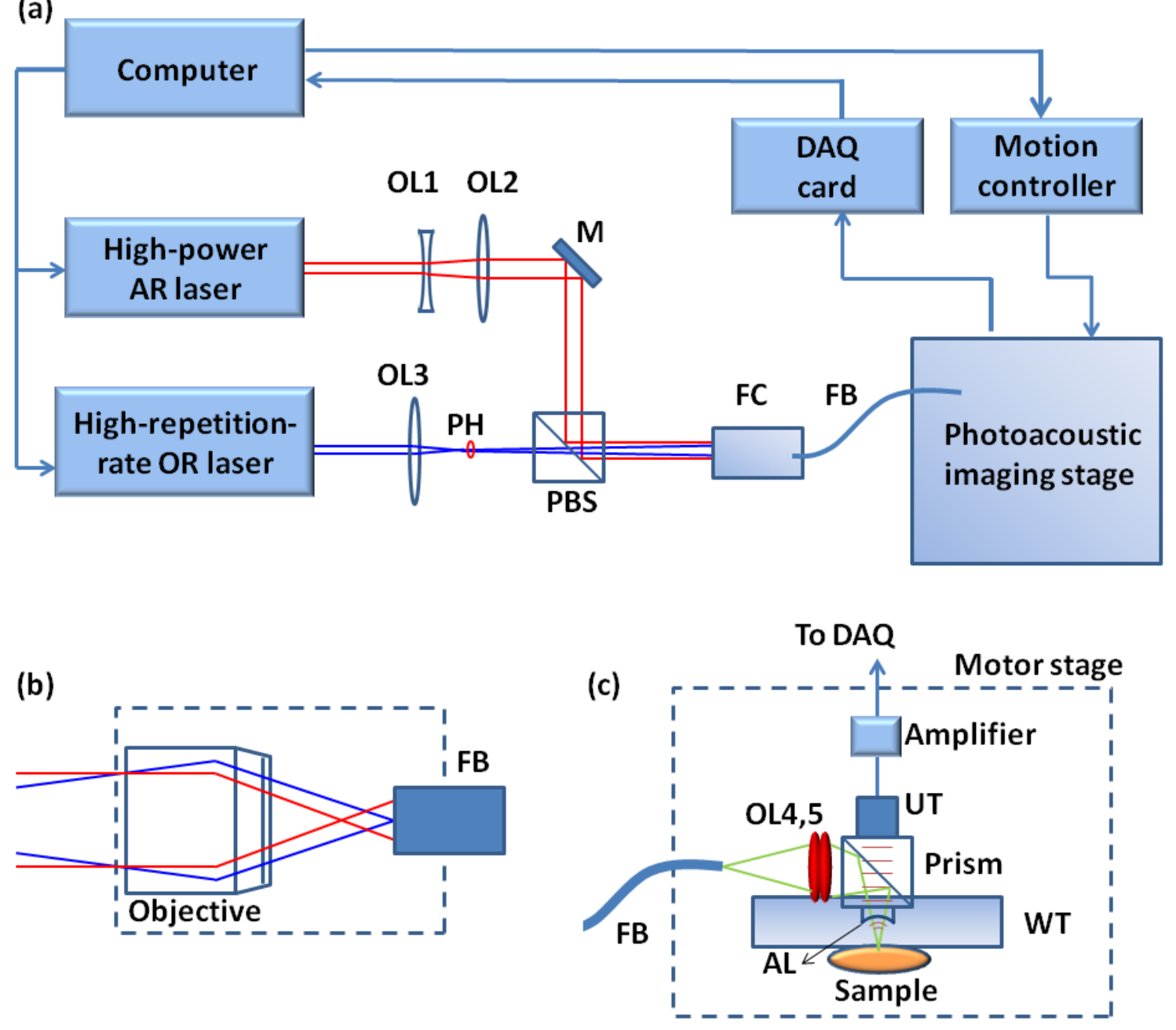

Figure 1(a) Schematic of the combined AR and OR photoacoustic microscopy system. (b)The interior of the fiber coupler. (c).Detailed design of the photoacoustic imaging stage. All images are side view images. AL, acoustic lens; DAQ,data acquisition; FB, fiber bundle; FC, fiber coupler; M, mirror; OL, optical lens; PBS, polarizing beamsplitter; PH, pinhole; UT, ultrasound transducer; WT, water tank.

cube's high extinction ratio $\left(T_{p}: T_{s}>1000: 1\right.$ for transmission;, $R_{s}: R_{p} \sim 100: 1$ for reflection; Subscript s: s-polarized beam, i.e., perpendicularly polarized AR beam; Subscript p: p-polarized beam, i.e., parallel polarized OR beam), most of the beam energy goes in the direction of a fiber coupler (F-91-C1, Newport, with a 10X magnification objective lens). After attenuation by a variable neutral density filter (NDC-100C-2, Thorlabs, not shown), the two beams pass into the fiber coupler. A fiber bundle is used to achieve switchable AR and OR illumination (IGN037/10, Sumitomo, core number: 10,000, single core diameter: $2.0 \mu \mathrm{m}$, entire core area diameter $\sim 350 \mu \mathrm{m}$ ). A single core is used to deliver OR light in order to achieve a small spot size and hence high lateral resolution, while the entire core area is used to deliver more energy for AR illumination. To focus the OR beam onto a single core of the fiber bundle, the pinhole is located $20 \mathrm{~cm}$ from the fiber coupler. The fiber coupler also focuses the collimated $\mathrm{AR}$ beam over the entire core area. The tip of the fiber bundle is aligned at the focus of the OR beam, which is slightly away from the focus of the AR beam [shown in Fig. 1(b)], making it match the entire core area at the fiber tip.

The other end of the fiber bundle is mounted to a photoacoustic imaging stage [Fig 1(c)]. The laser beams emerging from the fiber bundle pass through two identical optical condenser lenses, reflect off the aluminumcoated hypotenuse of a prism, and then focused into the sample. The generated photoacoustic signal is collected by 
the acoustic lens, which is in confocal arrangement with focused laser beam, and then received by a $50 \mathrm{MHz}$ ultrasound transducer (V214, Olympus NDT). The electrical signal from the ultrasonic transducer is amplified, digitized and analyzed by a personal computer, which also synchronizes the motion controller with the DAQ card and the lasers. A more detailed description on fast voice-coil scanning can be found in our previous publication [10]. Since the photoacoustic probe weighs less than $40 \mathrm{~g}$ as our previous one, the fast scanning ability is maintained in the combined OR/AR-PAM system.

\section{RESULTS}

\subsection{System resolution}

To evaluate the performance of the system, the spatial resolutions for both $\mathrm{OR}$ and $\mathrm{AR}$ imaging were measured. A resolution target (1951 USAF Hi-Resolution Targets, Edmund Optics) was imaged in water with only OR-PAM. The system can resolve element 6 in group 8 [arrow in Fig. 2(a)], with 456 line pairs per millimeter, which translates into an OR imaging lateral resolution finer than $2.2 \mu \mathrm{m}$. The discrepancy from the theoretical resolution of $1.4 \mu \mathrm{m}$ (NA: 0.2 , wavelength: $532 \mathrm{~nm}$ ) may be attributed to optical aberration and higher-order modes of the optical fiber. The lateral resolution of the AR imaging was evaluated by imaging a $6 \mu$ m-diameter carbon fiber as an approximate line target. The lateral full width at half-maximum (FWHM) is $40 \mu \mathrm{m}$, as shown in Fig. 2(b). Since the axial resolution is determined by the acoustic parameters only, the OR and AR imaging share the same axial resolution. The aixal resolution was estimated by the shift-and-sum method [11]. It simulates the situation when two line sources are positioned close to each other. The axial line spread function (LSF), its shift (16 um apart) and the envelope of their sum are shown in Fig. 2(c). The LSF is acquired by averaging 300 measurements, so it can be treated as a noise-free result. The envelope of the sum can be regarded as the envelope of the PA signal when two line sources are $16 \mu \mathrm{m}$ apart in the axial direction. The fact that the envelope has two peaks means two lines can be resolved. However, the actual resolving ability is always limited by noise. Here we define the contrast as
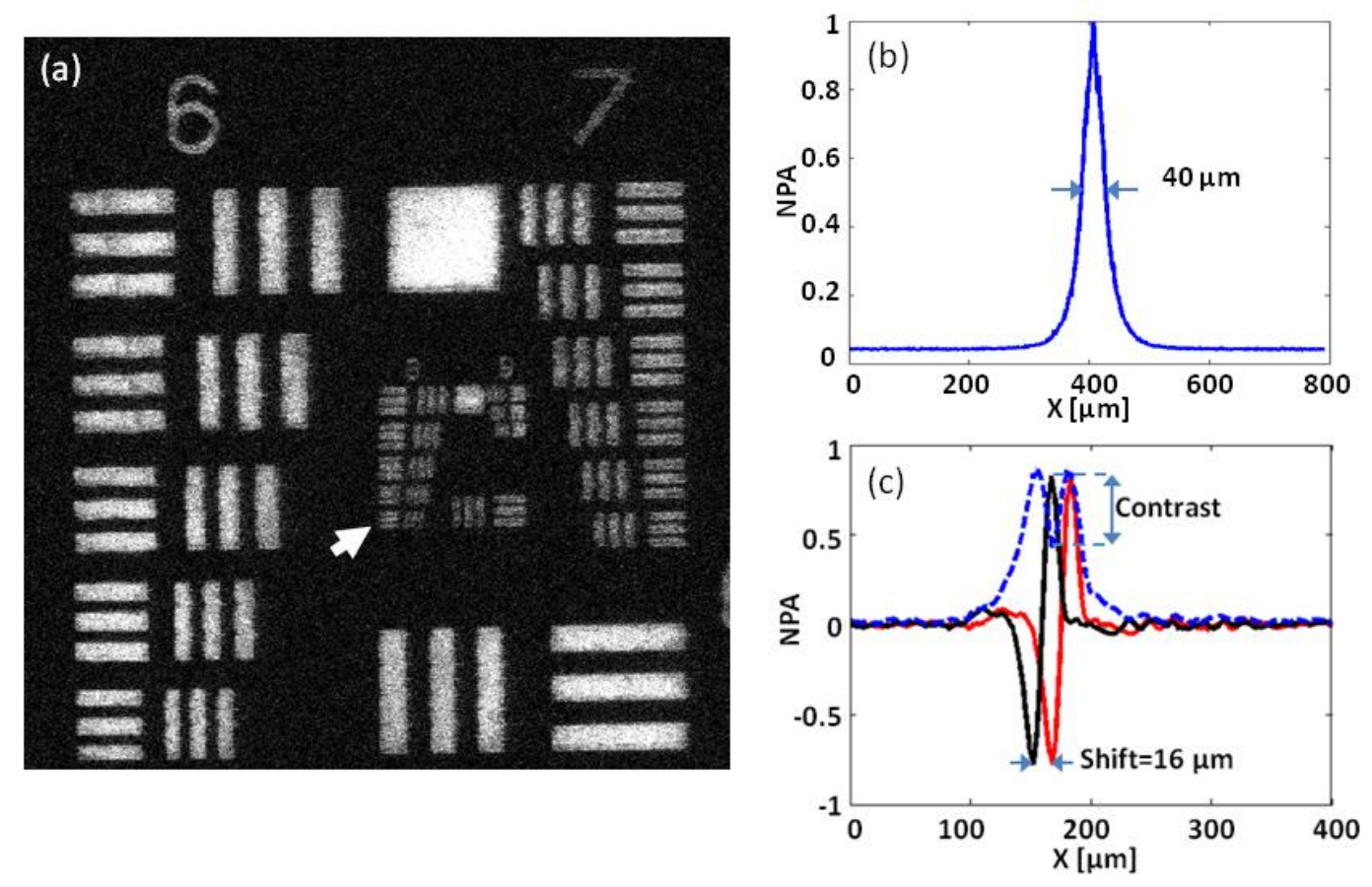

Figure 2 (a) OR-PAM image of a resolution test target (b) Lateral LSF of the AR-PAM. (c) Axial LSF (black solid curve), its shift (red solid curve) and the envelope of their sum (blue dashed curve). NPA, normalized photoacoustic amplitude. 
the signal difference between the bottom of the dip and the peak of the envelope, and the noise as the standard deviation of the signal noise in one single measurement. The resolution based on the shift-and-sum method is $\sim 16$ $\mu \mathrm{m}$ at a contrast-to-noise ratio of 2.0 .

\subsection{In vivo mouse imaging}

The system enables simultaneous OR and AR imaging. The two lasers are triggered alternately, each firing at its own pulse repetition rate, and the OR laser fires with less energy than the AR laser. For highest resolution, the OR laser is triggered with every step of the motor scanning, while the AR laser is triggered only once in ten steps. Thus, the OR and AR images can be acquired simultaneously in one single scan, with step sizes of $2 \mu \mathrm{m}$ and 20 $\mu \mathrm{m}$ and laser repetition rates of $20 \mathrm{kHz}$ and $2 \mathrm{kHz}$, respectively. In vivo mouse imaging was performed by the combined system. All experimental animal procedures were carried out in conformity with the laboratory protocol approved by the Animal Studies Committee at Washington University in Saint Louis. The mouse (Athymic Nude Mice, Harlan) was anaesthetized by isoflurane and positioned on an animal platform. The ear of a nude mouse was imaged by both AR-PAM and OR-PAM as shown in Fig. 3. The OR image shows fewer blood vessels due to the penetration depth but better image due to the resolution. The arrows in Fig 3(a) and Fig 3(b) indicate a deep vessel that AR-PAM can image, but OR-PAM was unable to image. Fig 3(c) and Fig 3(d) show the cross-sectional (B-scan) image located at dashed line position in Fig 3(a) and Fig 3(b). They clearly show that AR-PAM can image deeper than OR-PAM. As pointed by the small arrwos in Fig 3(c) and Fig 3(d), AR-PAM can image the blood vessels in both upper and bottom layers of the mouse ear, hower OR-PAM can only image the upper layer. The large arrows mark the same vessel which is marked in Fig 3(a) and Fig 3(b). It confirms that OR-PAM is unable to image it due to its shallow penetration depth.

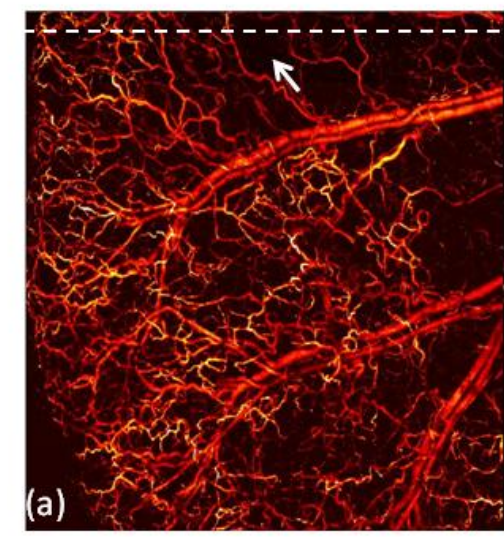

$1 \mathrm{~mm}$

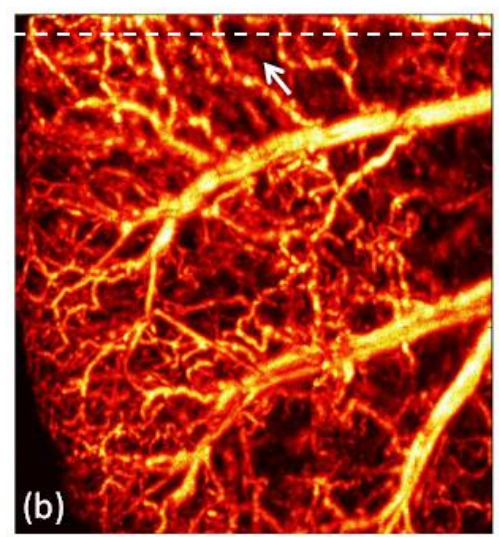

$1 \mathrm{~mm}$
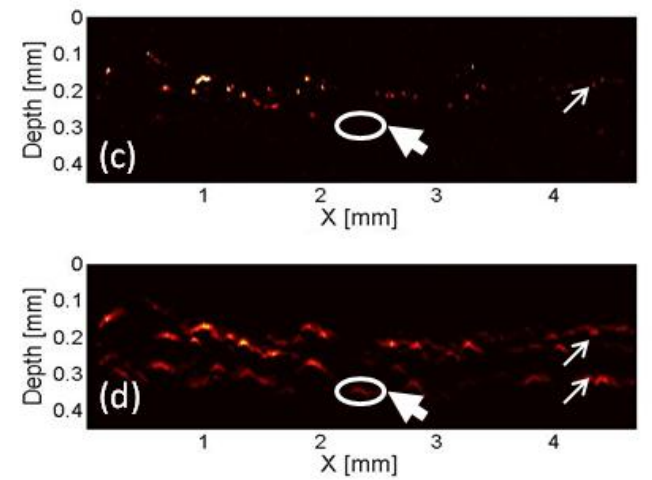

Figure 3 (a) OR-PAM image of the mouse ear. (b) AR-PAM image of the mouse ear. The arrows in (a) and (b) indicate a vessel which was successfully imaged by AR-PAM only. (c) Cross-sectional (B-scan) OR image at the position marked by dashed line in (a). The small arrow shows the upper layer of vessels. (d) Cross-sectional (B-scan) AR image at the position marked by dashed line in (b). The small arrows show both the upper and bottom layers of vessels. The big arrows in (c) and (d) indicate the same vessel as in (a) and (b). 
A region on the back of a nude mouse was imaged in vivo as well. Fig 4(a) and Fig 4(b) show the OR and AR image respectively. Fig 4 (d) and Fig 4 (e) show the cross-sectional image marked by the dash line in Fig 4(a) and Fig 4(b). Time-gain compensation was applied to show the deep signals better. AR-PAM is able to get signals up to $\sim 1.5 \mathrm{~mm}$ as the big arrow in Fig 4(e). Since AR-PAM does not have advantages over OR-PAM at shallow depth, we remove the surface signal (0-0.5 mm depth) in the AR-PAM image [dash line area in Fig 4(e)], and the new image is shown in Fig 4(c). It clearly shows large deep vessel which is different from the vessels shown in Fig 4(a). A merged image with shallow OR image and deep AR image is shown in Fig 4(f). It demestrates complementary information which the combined system can provide.
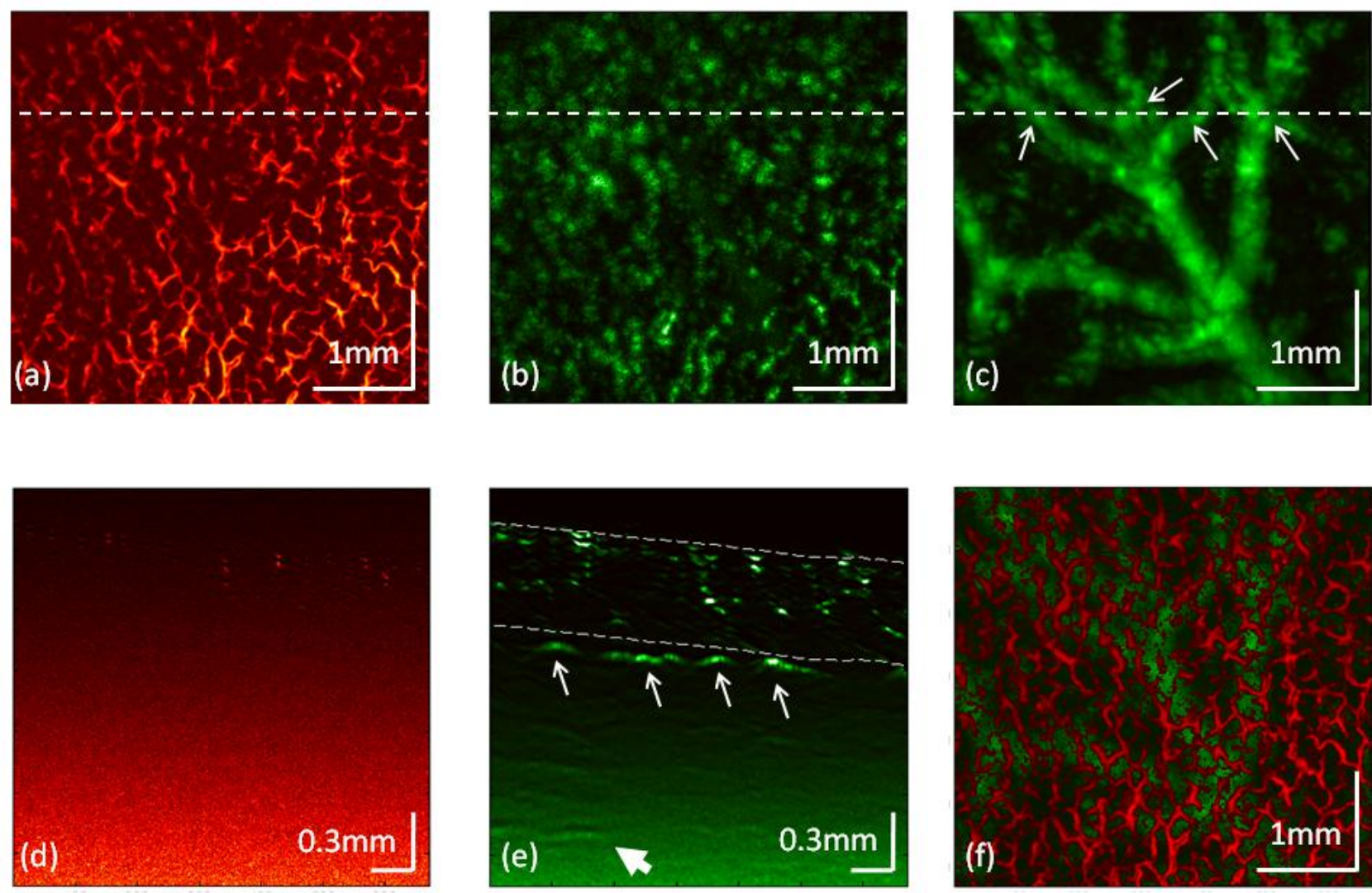

Figure 4 In vivo image of mouse skin: (a) Maximum amplitude projection (MAP) image by OR-PAM (b) MAP image by AR-PAM (c) MAP image by AR-PAM after the top $0.5 \mathrm{~mm}$ surface signal removed (d) (e) Crosssectional image at the dashed line marked position in (a) and (b). The large arrow in (e) indicates that AR-PAM could receive signal up to $1.5 \mathrm{~mm}$ depth in one single scan. Small arrows in (c) and (e) indicate the corresponding blood vessels. Dashed line shows the shallow signal, which is removed in (c). (f) Overlaid image of OR image and surface-removed AR image.

\section{CONCLUSION AND DISCUSSION}

To our knowledge, this is the first system that can perform OR and AR photoacoustic imaging simultaneously. The method we present here requires only modest modifications to current widely used PAM systems. Not limited to the two illumination choices introduced in this paper, the fiber bundle allows a wide range of illumination spot sizes, from one single core to the entire core area, and optical scanning among the cores is also possible [12]. A double clad [13] or multiple clad fiber may be applied to provide flexible illumination spot sizes as well. In this report, we demonstrate that the combined system can get complementary information when we image the sample by OR- and AR-PAM focused at the same depth, and remove the surface signal acquired by AR-PAM. This mode is 
especially beneficial in the imaging situations, when scans at the same depth are necessary. For example, scans by OR- and AR-PAM at the same depth are very useful for tumor imaging, since they can provide both strong penetrating capability for imaging a melanin-containing tumor and high-resolution capability for imaging the blood vessels around the tumor. Another mode is also possible, by separating the optical focus and the acoustic focus. That mode is suitable for getting OR- and AR-PAM images at different depths, and surface signal remove is not needed [14].

In summary, the combined system has successfully achieved switchable spatial resolutions and maximum imaging depths using an optical fiber bundle. This new combined OR/AR-PAM system can bring multiscale imaging capability to many applications, such as anatomic imaging and label-free measurement of oxygen saturation.

\section{ACKNOWLEDGEMENTS}

This work was sponsored by National Institutes of Health (NIH) grants R43 HL106855, R01 EB000712, R01 EB008085, R01 CA134539, U54 CA136398, R01 CA157277, R01 CA159959, as well as the Cancer Frontier Fund. L. V. Wang has a financial interest in Microphotoacoustics, Inc., and Endra, Inc., which did not support this work. K. Maslov has a financial interest in Microphotoacoustics, Inc. which did not support this work. The authors appreciate Prof. James Ballard's close reading of the manuscript.

\section{REFERENCES}

[1] Wang, L. V. and Hu, S., "Photoacoustic tomography: in vivo imaging from organelles to organs," Science 335, 1458-1462 (2012).

[2] Beard, P., "Biomedical photoacoustic imaging," Interface Focus 1, 602-631 (2011).

[3] Zhang, H. F., Maslov, K., Stoica, G., and Wang, L. V., "Functional photoacoustic microscopy for high-resolution and noninvasive in vivo imaging," Nat Biotechnol 24, 848-851 (2006).

[4] Liao, L. D., Lin, C. T., Shih, Y. Y. I., Duong, T. Q., Lai, H. Y., Wang, P. H., Wu, R., Tsang, S., Chang, J. Y., and Li, M. L., "Transcranial imaging of functional cerebral hemodynamic changes in single blood vessels using in vivo photoacoustic microscopy," J. Cereb. Blood Flow Metab. 32, 938-951 (2012).

[5] Maslov, K., Zhang, H. F., Hu, S., and Wang, L. V., "Optical-resolution photoacoustic microscopy for in vivo imaging of single capillaries," Opt. Lett. 33, 929-931 (2008).

[6] Xie, Z., Jiao, S., Zhang, H. F., and Puliafito, C. A., "Laser-scanning optical-resolution photoacoustic microscopy," Opt. Lett. 34, 1771-1773 (2009).

[7] Hu, S., Maslov, K., and Wang, L. V., "Second-generation optical-resolution photoacoustic microscopy with improved sensitivity and speed," Optics Letters 36, 1134-1136 (2011).

[8] Favazza, C. P., Hu, S., Huang, V., Jassim, O., Cornelius, L. A., and Wang, L. V., "In vivo multiscale photoacoustic microscopy of human skin," Proc. SPIE 7899, 789946 (2011).

[9] Cai, X., Paratala, B. S., Hu, S., Sitharaman, B., and Wang, L. V., "Multiscale photoacoustic microscopy of singlewalled carbon nanotube-incorporated tissue engineering scaffolds," Tissue Eng. Part C: Methods 18, 310-317 (2011).

[10] Wang, L., Maslov, K., Yao, J., Rao, B., and Wang, L. V., "Fast voice-coil scanning optical-resolution photoacoustic microscopy," Opt. Lett. 36, 139-141 (2011).

[11] Ku, G., Maslov, K., Li, L., and Wang, L. V., "Photoacoustic microscopy with 2- $\mu \mathrm{m}$ transverse resolution," J Biomed Opt 15, 021302 (2010).

[12] Hajireza, P., Shi, W., and Zemp, R. J., "Label-free in vivo fiber-based optical-resolution photoacoustic microscopy," Opt. Lett. 36, 4107-4109 (2011).

[13] Ryu, S. Y., Choi, H. Y., Na, J., Choi, E. S., and Lee, B. H., "Combined system of optical coherence tomography and fluorescence spectroscopy based on double-cladding fiber," Opt. Lett. 33, 2347-2349 (2008).

[14] Xing, W., Wang, L., Maslov, K., and Wang, L. V., "Integrated optical- and acoustic-resolution photoacoustic microscopy based on an optical fiber bundle," Opt. Lett. 38, 52-54 (2013) 